\title{
Optimal sizing of a parallel PHEV powertrain
}

\author{
Mitra Pourabdollah, Nikolce Murgovski, Anders Grauers and Bo Egardt \\ Chalmers University of Technology, Gothenburg, Sweden \\ mitrap, nikolce.murgovski, anders.grauers, bo.egardt@chalmers.se
}

\begin{abstract}
This work introduces a novel method for the simultaneous optimization of energy management and powertrain components sizing of a parallel plug-in hybrid electric vehicle. The problem is formulated as a convex optimization problem to minimize an objective function, which is a weighted sum of operational and component costs. The former includes the consumed fossil fuel and electrical energy, whereas the latter includes the cost of battery, electric motor, and internal combustion engine. The powertrain model includes quadratic losses for the powertrain components. Moreover, the combustion engine and the electric motor losses are assumed to scale linearly with respect to the size and the losses of baseline components.

The result of the optimization is the variables of the global optimal energy management for every time instant and optimal component sizes. Due to the dependency of the result on the driving cycle, a long real-life cycle with its charging times is chosen to represent a general driving pattern. The method allows studying the effect of some performance requirements, namely acceleration, top speed, and all electric range, on the component sizes and total cost.
\end{abstract}

\section{INTRODUCTION}

Interest in the electrification of vehicles is growing as a result of the environmental benefits that can be achieved regarding emissions and fuel consumption. Hybrid electric vehicles (HEV) are the first generation of electrified vehicles that, in addition to an internal combustion engine (ICE), have an electric motor (EM) and electric energy storage. HEVs can improve fuel efficiency owing to the possibility of downsizing the engine, the ability to recover braking energy, the extra power control freedom gained by the two power sources, and the ability to stop the engine when idle. The next generation of electrified vehicles, plug-in hybrid electric vehicles (PHEV), have the additional ability to store energy from the electricity grid using large capacity batteries. The stored energy can propel the vehicle on short trips, thereby reducing vehicle dependency on petroleum and potentially $\mathrm{CO}_{2}$ emissions. The extent to which this can be achieved depends on the size of the battery and the driving habits of the vehicle owner. There is clearly a trade-off between improved fuel economy and battery cost (size). This is one of the many design trade-offs involved with PHEVs when taking into account both cost effectiveness and performance of the vehicle [1]. These trade-offs are highly sensitive to the varying prices of energy and components, and the driving and charging patterns. Finding the proper balance between different objectives is also related to customers' 
expectations. For example, if the vehicle were to be optimized for typical daily city driving, it would most likely not be able to deliver high performance, which is rarely needed, but can be attractive for many customers ${ }^{1}$.

The discussion above raises many questions regarding the design of a PHEV, for example:

- Is components' sizing mainly driven by performance requirements, such as all-electric range (AER), acceleration abilities and top speed, or is there a lot of freedom left to optimize the component sizes against the driving pattern?

- How much do the component costs and/or fuel consumption decrease when performance requirements are relaxed?

- Which performance requirements are cheaper to fulfill?

- What is the improvement in fuel economy when the vehicle utilizes a larger battery or EM?

The traditional approach to investigate these issues would be to consider many design candidates, each meeting a set of requirements, see e.g., in [3]. These candidates, would then, be evaluated by simulations to find the vehicle with the best overall trade-off between cost and performance.

One aspect of the (P)HEVs, which distinguishes them from conventional cars, is energy management, i.e., the control strategy that determines how the battery should be used (or, basically, how the power should be split between EM and ICE). Energy management affects both fuel economy and performance, which means that it should ideally be part of the design process in the same way as component sizing. One way of doing this is to (algorithmically) find the optimal energy management for each design candidate. Since real-time implementations of energy management strategies are not sought during this phase, Dynamic Programming (DP) is a natural candidate for finding the optimal energy management. One example of this approach is the work in [4].

A disadvantage of using DP to find the optimal energy management is that it has to be run many times during the optimization/evaluation of vehicle designs, resulting in a high computational burden. Therefore, it is common practice to instead apply a suitably parameterized strategy, which is often rule based. The problem of powertrain component sizing that relies on rule based control has been considered in [5], [6], [7], [8], [9], [10], and [11]. Usually the parameters are optimized simultaneously with the components' sizes. A drawback of the method is that the choice of control structure will influence the optimal component sizes; for example, in [12] the authors show that an HEV may require a battery with a larger capacity when applying a rule-based energy management strategy compared to a so-called blended strategy. Another approach is given in [13], where a rule based energy management is first applied to find optimal component sizes and then DP is used to refine the suboptimal rules for the vehicle with the obtained components' sizes.

A different approach is taken in [14] to simultaneously optimize both battery size and energy management for a plug-in hybrid bus. The idea is to cast the problem as a convex optimization problem based on quasi-static models. Convexity has two important implications: there is a unique optimum and fast, reliable solvers are available. This

\footnotetext{
${ }^{1}$ A survey conducted by Consumer Reports in [2] shows that half of the customers who want to buy a new car would sacrifice performance or vehicle size for better fuel economy and $38 \%$ are even willing to pay more in order to get lower fuel consumption.
} 
in turn means that it is feasible to include as optimization variables not only component sizes but also the complete control trajectory of the energy management system. This results in a large number of variables in the problem (easily tens of thousands). Despite this, the fast computations make it suitable to use very long driving cycles. Being able to handle long and varying driving cycles has potential benefits in giving solutions with better robustness to driving pattern variations. To remove the need for solving a mixed integer problem, the decisions on gear selection and engine on-off are made outside the convex optimization based on heuristics.

This paper is a continuation of the work in [14], extending the method to find the optimal sizes of battery, EM, and ICE for a parallel PHEV. In addition, we add different levels of performance requirements to the problem, and we study the effect of these constraints on component sizes and total cost. To clarify the basic ideas, an outline of the studied optimization problem is given as follows. The objective function to be minimized over a pre-specified driving cycle comprises of two parts. The first part reflects the cost of the key components of the vehicle, namely battery, EM, and ICE. This cost is the depreciation over the chosen driving cycle. The second part is the operational cost of fuel and electricity. The optimization is done over a long driving cycle, which includes opportunities for charging from the grid. The constraints are given by equations governing the power flow in the system and the component models, and by the maximum component ratings. Finally, the decision variables to be found by the optimization solver are the component sizes (battery, EM, and ICE) and the optimal energy management (torque split, battery current, braking, and charging at every time instant). Similarly as in [14], the decisions for the gears and engine on-off are made based on heuristics outside the convex optimization.

The aim of this work is to provide a tool that can be used during the initial exploratory phase of vehicle design. It allows a better understanding of the effects of components and energy prices, driving patterns, and performance requirements on the design of a PHEV. To illustrate how the method can be used, we show how the performance requirements affect the component sizes.

The paper is organized as follows. In Section II, an overall view of the convex optimization problem is given. The main ingredients are described and it is discussed how, e.g., the driving cycle and performance requirements are entered into the problem formulation. Section III continues with the convex formulation of the vehicle and component models; these models describe both the power flow and cost aspects. In Section IV, the mathematical formulation of the convex optimization problem is given in more detail. Some illustrative results from the sensitivity study are shown in Section V. Finally conclusions are drawn.

\section{Problem Statement}

The optimization problem that was briefly introduced in the Introduction will now be described in some more details. We will defer the mathematical details to Section III, after having introduced the component models. As shown in Fig. 1, for a vehicle model and a given driving cycle, we first find the gears and engine on-off decisions. Having these, in addition with the cost model and the performance requirements, a convex sub-problem is iteratively 


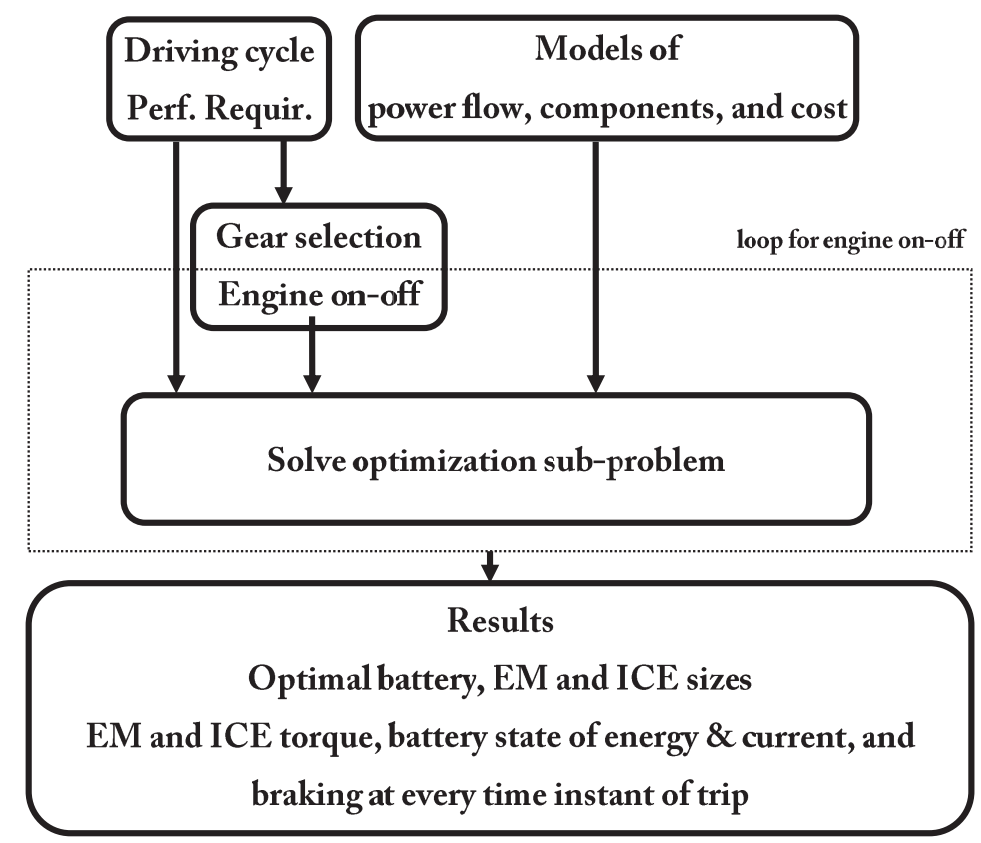

Fig. 1. Framework of the optimization problem. Gears and engine on-off are decided by heuristics and the rest of the optimization variables are obtained by iteratively solving a sub-problem.

solved to find the optimization parameters. The basic statement of the sub-problem is as follows

$$
\begin{aligned}
& \text { minimize } \text { cost } \\
& \text { with respect to }\left\{\begin{array}{l}
\text { component sizes } \\
\text { energy management } \\
\text { charging from grid, }
\end{array}\right. \\
& \text { subject to }\left\{\begin{array}{l}
\text { driving mission } \\
\text { performance requirements } \\
\text { selected gear and engine on-off } \\
\text { powertrain model } \\
\text { component limitations. }
\end{array}\right.
\end{aligned}
$$

Each of the ingredients of the optimization problem is described in the following subsections.

\section{A. Optimization cost}

The optimization cost to be minimized includes operational and component costs:

$$
\operatorname{cost}=\operatorname{cost}_{o p}+\operatorname{cost}_{\text {comp }} \text {. }
$$

The operational cost is the sum of the fuel and electricity costs over the given driving cycle. The fuel power, $P_{f}$, and charger power, $P_{g}$, are converted to an equivalent cost in EUR using energy prices $\rho_{f}$ for gasoline and $\rho_{e l}$ for 
TABLE I

Parameters for CAlculating costs

\begin{tabular}{ll}
\hline price of gasoline $\left(\rho_{f}\right)$ & $1.6 \mathrm{EUR} / \mathrm{L}$ \\
price of electricity $\left(\rho_{e l}\right)$ & $0.15 \mathrm{EUR} / \mathrm{kWh}$ \\
lower heating value $\left(\rho_{L H V}\right)$ & $42.8 \times 10^{6} \mathrm{~J} / \mathrm{L}$ \\
yearly interest rate $\left(p_{c}\right)$ & $\% 5$ \\
vehicle lifetime $\left(y_{v}\right)$ & 10 years \\
yearly traveled distance $(s)$ & $20000 \mathrm{~km}$ \\
\hline
\end{tabular}

electricity [15]. The operational cost calculated over a discretized driving cycle of $N$ sampling intervals of length $h(k)$, becomes

$$
\operatorname{cost}_{o p}=\frac{\rho_{f}}{\rho_{L H V}} \sum_{k=1}^{N} P_{f}(k) h(k)+\frac{\rho_{e l}}{1000 \cdot 3600} \sum_{k=1}^{N} \boldsymbol{P}_{\boldsymbol{g}}(\boldsymbol{k}) h(k) .
$$

where $\rho_{L H V}$ is the lower heating value of gasoline. The sampling interval $h(k)$ is time varying; it is equal to $1 \mathrm{~s}$ while driving and the whole parking time while charging. The detail is given later in Section III-B.

The component cost is the sum of the costs of battery, EM, and ICE; the remaining costs of the vehicle are independent of sizing and are therefore excluded from the problem. The component costs are calculated as the depreciation over the driving cycle, i.e., the proportion of the component costs given by the ratio between the length of the cycle, $d$, and the lifetime driving distance of the vehicle. Including a yearly interest rate of $p_{c}=5 \%$, the component cost is given by

$$
\operatorname{cost}_{\text {comp }}=\frac{d}{s y_{v}}\left(1+p_{c} \frac{y_{v}+1}{2}\right)\left(\operatorname{cost}_{b a t}+\operatorname{cost}_{E M}+\operatorname{cost}_{I C E}\right),
$$

where $y_{v}$ is the vehicle lifetime, and $s$ is the average traveled distance of the vehicle in one year. For the used driving cycle of length $700 \mathrm{~km}$, the depreciation is roughly 0.0045 of the total cost. The parameters in (2) and (3) are given in Table I.

\section{B. Optimization variables}

The decision variables of the optimization problem include, firstly, the component sizes $\boldsymbol{s}_{\boldsymbol{b a t}}, \boldsymbol{s}_{\boldsymbol{E} \boldsymbol{M}}$, and $\boldsymbol{s}_{\boldsymbol{I C E}}$, which are all dimensionless scaling parameters. The second group consists of optimization variables, which are related to the energy management and are determined for every time instant. These variables are the torques $\boldsymbol{T}_{\boldsymbol{E M}}(\boldsymbol{k})$ and $\boldsymbol{T}_{I C E}(k)$, battery current, $\tilde{\boldsymbol{i}}(\boldsymbol{k})$, battery state of energy, $\boldsymbol{E}_{\boldsymbol{b}}(\boldsymbol{k})$, grid power, $\boldsymbol{P}_{\boldsymbol{g}}(\boldsymbol{k})$, and braking power, $\boldsymbol{P}_{\boldsymbol{b r k}}(\boldsymbol{k})$. The optimization variables are marked in bold in the paper.

\section{Driving cycle and charging from grid}

The optimization gives the optimal component sizes and energy management for a certain driving cycle. Ideally, the optimization should be performed over the vehicle's lifetime driving patterns. However, this is not possible due to driving uncertainties and limited computational resources. Therefore, a shorter driving cycle including typical 
TABLE II

PARAMETERS OF THE CYCLE

\begin{tabular}{ll}
\hline number of days & 20 \\
number of trips & 67 \\
number of charging events $\left(N_{c}\right)$ & 30 \\
distance & $712.9 \mathrm{~km}$ \\
number of samples $(\mathrm{N})$ & $60000 \mathrm{~s}$ \\
max. speed & $120.3 \mathrm{kph}$ \\
average speed & $42.8 \mathrm{kph}$ \\
max. acceleration & $3.3 \mathrm{~m} / \mathrm{s}^{2}$ \\
max. deceleration & $-4.3 \mathrm{~m} / \mathrm{s}^{2}$ \\
\hline
\end{tabular}

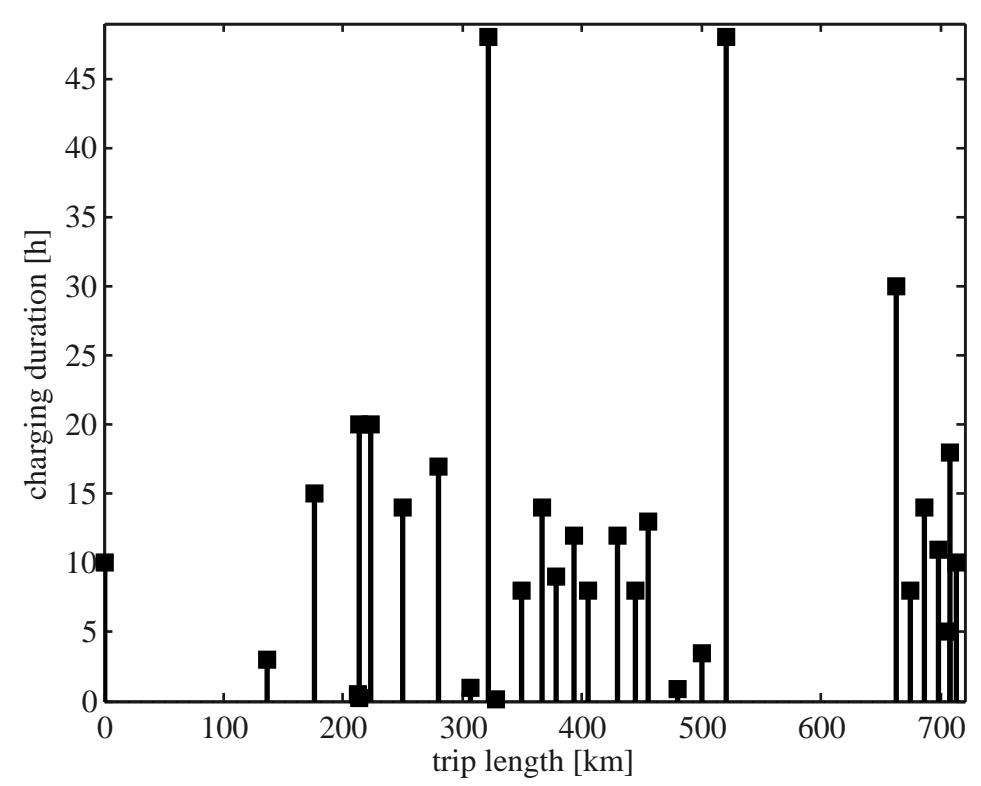

Fig. 2. Charging duration at each occasion, the vehicle can only be charged at specific locations.

driving patterns that reflect real-life driving is chosen. The driving cycle specifies the vehicle velocity, $v(k)$, and possibly the road inclination, $\beta(k)$, at discrete time instants, and charging times between the trips.

The driving data used in the examples are provided by ETC $^{2}$ from a plug-in hybrid Volvo V70 and includes a long sequence of real-life driving data. We assume that the vehicle is driven on a horizontal road. The parameters of the cycle are given in Table II. At certain parking locations along the driving cycle, the vehicle has the possibility to charge from the grid. parking durations (with charging opportunities) are taken from real data and it is assumed that the vehicle can use the entire parking time, $\Delta t_{c}$, for charging; this is shown in Fig. 2. Charging is presented in more detail in Section III-B.

${ }^{2}$ ETC Battery and FuelCells Sweden AB 


\section{Performance requirements}

A long driving cycle can reflect real-life driving, but might not include situations that require high performance. However, high performance is still considered an important vehicle attribute by many drivers. There are basically two ways to incorporate such performance requirements into the optimization formulation; the first one is to include explicit constraints on the component sizes, the second is to indirectly impose constraints by including some extreme driving conditions into the driving cycle. Both techniques will be used in our problem to include the following three performance parameters: all electric range (AER), charge sustaining top speed, and acceleration at different speeds. Each of these will be described in the following.

a) All electric range (AER): The AER of a vehicle is the distance driven using only electric power from the battery. The AER is generally measured in kilometers (or miles), but it requires a reference standard driving cycle, here the New European Driving Cycle (NEDC). For the studied vehicle, a battery capacity of $1 \mathrm{kWh}$ corresponds approximately to $3.8 \mathrm{~km}$ AER. In the optimization, the required AER is translated to a lower bound on the size of the battery.

b) Top speed: The charge sustaining top speed $v_{\max }$ is the highest steady-state speed with the traction force provided only by the ICE. This requirement gives a lower bound on the size of the ICE.

c) Acceleration: The requirements on acceleration at different speeds (or, equivalently, requirements on constant speed at different road gradients) can be fulfilled by different combinations of ICE and EM sizes. Therefore, to include this constraint, the acceleration requirement is converted into a so-called performance cycle, which is appended to the real-life driving cycle. The starting point is to notice that the requirements can be represented in a single plot of normalized net traction force, $F_{\text {norm }}(v)$ as a function of speed $v$. The normalized net force ${ }^{3}$ is simply the force needed to give the vehicle the required acceleration (or ascent capability), divided by mass:

$$
F_{\text {norm }}=a+g \sin (\beta)
$$

where $a$ is the vehicle acceleration, $g$ is the gravitational acceleration, and $\beta$ is the road inclination. The expression in (4) shows for example that a powertrain capable of producing a normalized net force of $F_{\text {norm }}=1 \mathrm{~N} / \mathrm{kg}$ will be able to accelerate with $1 \mathrm{~m} / \mathrm{s}^{2}$ on a flat road, or maintaining constant speed on a road with about $10 \%$ gradient. Therefore, specifying $F_{\text {norm }}$ is a reasonable way of specifying powertrain performance.

The baseline requirement of $F_{n o r m}$ to be used in our examples is taken from a Volvo C30 electric and is shown in Fig. 3. In this figure, we also plot different levels of performance requirements, obtained by multiplying the baseline curve by a coefficient $\alpha_{p}$, and changing the top speed. Finally, we also plot the accelerations from the real-life driving cycle (indicated by black dot markers) and the performance cycle (indicated by gray dot markers). The performance cycles, shown in Fig. 4, include speeds from zero to the top speed, with accelerations interpolated from the curves in Fig. 3 for different values of $\alpha_{p}$ and $v_{\max }$. As can be seen in Fig. 3, the performance requirements are more power demanding compared to the typical driving cycles. For example, out of 60000 acceleration points

\footnotetext{
${ }^{3}$ The total traction force also includes friction and air resistance effects.
} 


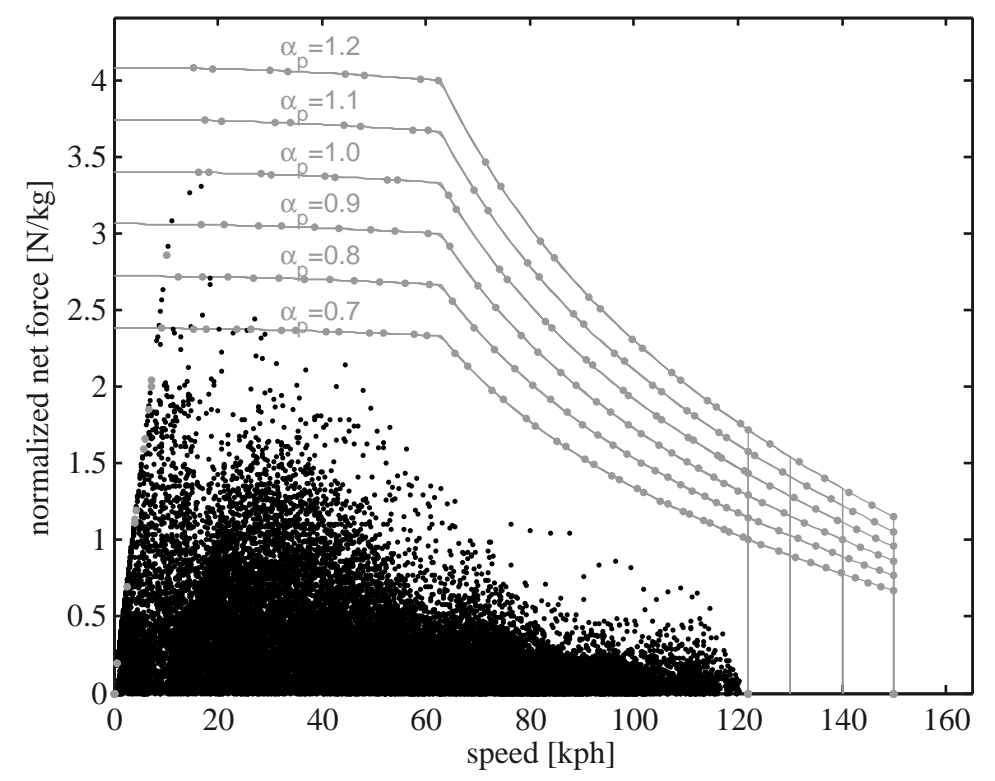

Fig. 3. Acceleration requirements (solid curves), acceleration of different performance driving cycles (gray dots), acceleration of the real-life driving cycle (black dots) vs. speed.

of the real-life driving cycle, at only 13 points the demanded acceleration is higher than the $70 \%$ of the baseline acceleration requirement $\left(\alpha_{p}=0.7\right)$. For acceleration requirement with $\alpha_{p}=0.8$ and $\alpha_{p}=0.9$, the numbers are 4 and 3 , respectively. If at any time instant in the simulations the acceleration is higher than the one from the requirements, we lower it by time stretching. The effect of this action on the results is very small since the number of affected points is low.

\section{E. Gear selection}

In general, gear shifting strategies that are functions of unknown optimization variables, such as torque or efficiency, may lead to a non-convex problem. The alternative is to decide gear, $\gamma(k)$, outside the convex optimization from a speed dependent hysteresis model applied on the known driving profile [18]. However, this strategy highly influences the optimal size of the ICE since it does not consider the engine peak power. Therefore, we modify it so that the gear strategy depends also on power; as a result, the gear shifting happens at higher speeds for high acceleration demands. The detail of this strategy is provided in appendix A.

\section{F. Powertrain constraints and components limitations}

The constraints in the optimization problem include also the equations governing the power flow in the system, the component models, limitations of the components (e.g., current, torque) and constraints on the component sizes. This will be explained in detail in the following section. 


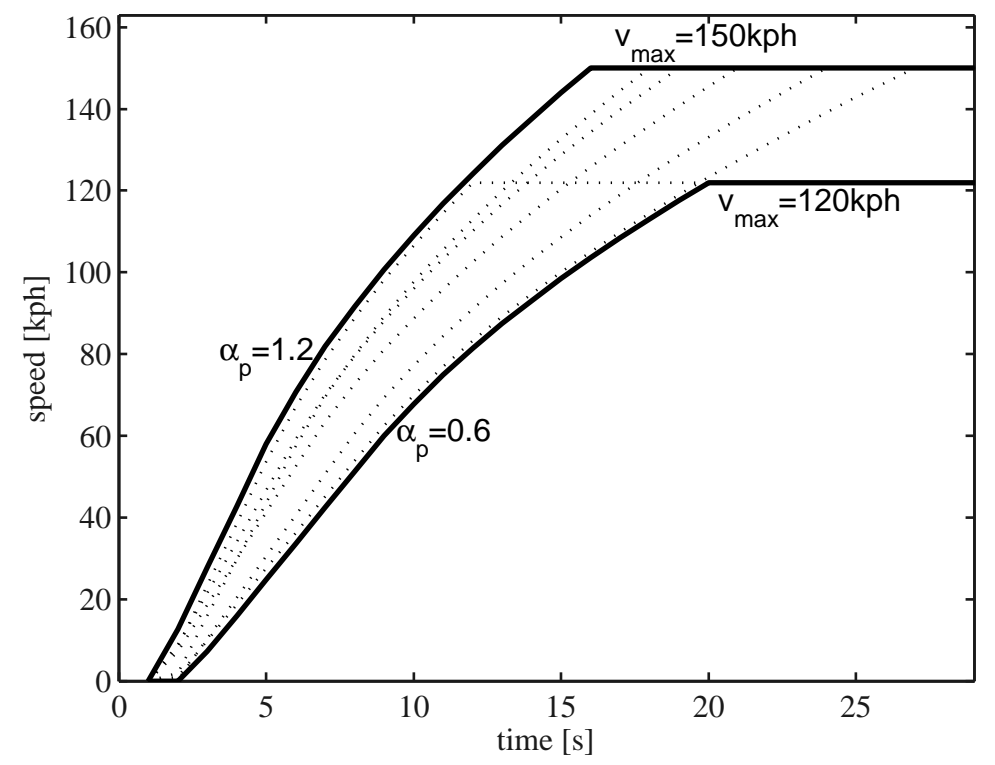

Fig. 4. Performance cycles constructed from the acceleration and top speed requirements, used to force the optimization to fulfill the requirements.

\section{Modeling DetaILS}

Modeling of the powertrain and its components is a crucial step of the presented method, since convexity has to be guaranteed. This section presents the powertrain model, the gear shifting strategy, and the component models. Particular attention is given to a novel method to describe the power losses of EM and fuel power of ICE with convex functions ${ }^{4}$, including the scaling of size. In the last part, the cost and mass models of these components are also presented.

\section{A. Powertrain model}

As depicted in Fig. 5, the studied PHEV includes a powertrain with parallel topology, where both ICE and EM are mechanically linked to the drive train and can propel the wheels. From the drive cycle, velocity $v(k)$, acceleration $a(k)$, and road slope $\beta(k)$, are obtained at discrete time instants $k$. The required traction force $F_{t}(k)$ can then be calculated as [17]

$$
F_{t}(k)=\frac{c_{d} A_{f} \rho v(k)^{2}}{2}+m_{t o t} g c_{r} \cos (\beta(k))+m_{t o t} g \sin (\beta(k))+m_{t o t} a(k)
$$

where $m_{t o t}, A_{f}, c_{d}, \rho, g$, and $c_{r}$ are the total vehicle mass, frontal area, air drag coefficient, air density, gravitational acceleration, and rolling resistance coefficient, respectively (details on the computation of $m_{\text {tot }}$ are given in Section III-E). It follows that the powertrain has to supply the demanded power $P_{\text {dem }}(k)=F_{t}(k) v(k)$, which is subject to

\footnotetext{
${ }^{4}$ A convex function satisfies $f(\lambda x+(1-\lambda) y) \leq \lambda f(x)+(1-\lambda) f(y)$ for all $x, y \in \mathbf{R}$ with $0 \leq \lambda \leq 1$ (see [16]).
} 


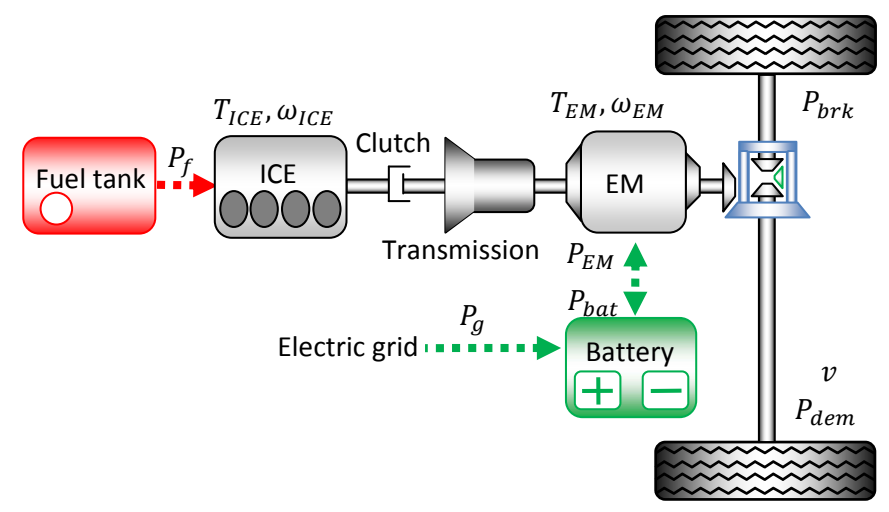

Fig. 5. Parallel PHEV configuration (solid lines: mechanical link, and dashed lines: electrical links).

the following mechanical and electrical power balance equations of the powertrain

$$
\begin{gathered}
P_{d e m}(k)+\boldsymbol{P}_{\boldsymbol{b r k}}(\boldsymbol{k})=\boldsymbol{T}_{\boldsymbol{E M}}(\boldsymbol{k}) \omega_{E M}(k)+e_{o n}(k) \boldsymbol{T}_{\boldsymbol{I C E}}(\boldsymbol{k}) \omega_{I C E}(k) \eta(k) \\
\omega_{E M}(k) \boldsymbol{T}_{\boldsymbol{E M}}(\boldsymbol{k})+P_{E M, l o s s}(k)+P_{a u x}(k)=P_{b a t}(k)+\boldsymbol{P}_{\boldsymbol{g}}(\boldsymbol{k}) \eta_{g},
\end{gathered}
$$

where $\boldsymbol{P}_{\boldsymbol{b r k}}(\boldsymbol{k})$ is the power dissipated at the friction brakes; $\boldsymbol{T}_{\boldsymbol{E} \boldsymbol{M}}(\boldsymbol{k}), \omega_{E M}(k)$, and $\boldsymbol{P}_{E M, l o s s}(k)$ are the torque, speed and power losses of the EM, respectively; $\boldsymbol{T}_{\boldsymbol{I C E}}(k)$, and $\omega_{I C E}(k)$ are the torque and speed of the ICE; $P_{a u x}(k)$ is the electrical power used by auxiliary devices $\left(P_{a u x}(k)\right.$ is assumed to be equal to $500 \mathrm{~W}$ when driving; $P_{b a t}(k)$ is the battery power; $\boldsymbol{P}_{\boldsymbol{g}}(\boldsymbol{k})$ is the grid power (including the losses) which is nonzero only at charging times and $\eta_{g}$ is the charger efficiency which is assumed to be constant, for simplicity. It is also assumed that the EM and auxiliaries are turned off during charging. The decision variable, $e_{o n}(k)$, is used to turn the ICE on or off and $\eta(k)$ is the transmission efficiency which depends on the choice of gear. For simplicity, the losses in the power electronics are included in the EM losses and the rotational inertia (summation of the inertia of the wheels, the differential, the EM, and the ICE) are neglected in the models. The vehicle parameters are given in Table III.

At each time instant of the driving cycle, the gear $\gamma(k)$ is selected based on the vehicle speed and demanded power of the powertrain with the baseline components. Details are given in the next subsection. Knowing the gear and the vehicle speed, the angular speeds $\omega_{E M}(k)$ and $\omega_{I C E}(k)$ can be calculated as

$$
\begin{aligned}
& \omega_{E M}(k)=r_{E M} \frac{r_{f g}}{r_{w}} v(k), \\
& \omega_{I C E}(k)=r_{\gamma}(\gamma(k)) \frac{r_{f g}}{r_{w}} v(k),
\end{aligned}
$$

where $r_{w}, r_{f g}, r_{E M}$ and $r_{\gamma}(\gamma(k))$ are the wheel radius, ratio of the final gear (differential), EM reduction gear, and ratio of the transmission gear, respectively [17]. The model does not allow any slip in the clutch; therefore, the vehicle is propelled by the EM at low speeds (typically when starting from stand still). 
TABLE III

VeHicLE PARAMETERS

\begin{tabular}{ll}
\hline \hline parameter & value \\
\hline baseline mass $(\mathrm{m})$ & $1600 \mathrm{~kg}$ \\
glider mass $\left(m_{g}\right)$ & $1280 \mathrm{~kg}$ \\
frontal area $\left(A_{f}\right)$ & $2.37 \mathrm{~m}^{2}$ \\
rolling resistance $\left(c_{r}\right)$ & 0.009 \\
aerodynamic drag coefficient $\left(c_{d}\right)$ & 0.33 \\
air density $(\rho)$ & $1.293 \mathrm{~kg} / \mathrm{m}^{3}$ \\
wheel radius $\left(r_{w}\right)$ & $0.3 \mathrm{~m}$ \\
ratio of the final gear $\left(r_{f g}\right)$ & 4.2 \\
EM reduction gear $\left(r_{E M}\right)$ & 2 \\
\hline
\end{tabular}

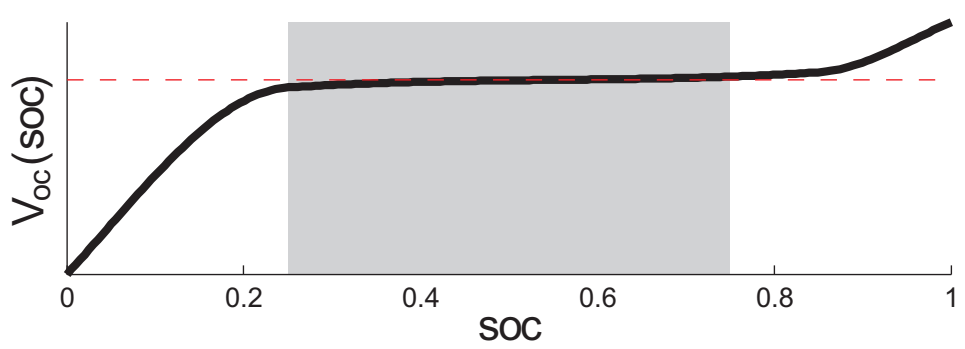

Fig. 6. Model of the battery open circuit voltage. The original model and the approximation are represented by the solid line and the dashed line. The gray area shows the allowed SoC operating region.

\section{B. Battery}

The battery consists of $\boldsymbol{s}_{\text {bat }}=n_{s} \cdot n_{p}$ identical cells, where $n_{s}$ is the number of cells in a string connected in series and $n_{p}$ is the number of strings connected in parallel. Considering the battery cell model as an open circuit voltage, $V_{o c}$, in series with a constant internal resistance, $R$ [17], the terminal power, $P_{b a t}$, and the stored energy of the battery, $E_{b}$, can be calculated as

$$
\begin{gathered}
P_{b a t}(k)=\boldsymbol{s}_{\boldsymbol{b a t}}\left(V_{o c} i(k)-R i^{2}(k)\right)=V_{o c} \tilde{\boldsymbol{i}}(\boldsymbol{k})-R \frac{\tilde{\boldsymbol{i}}^{2}(\boldsymbol{k})}{\boldsymbol{s}_{\boldsymbol{b a t}}} \\
\boldsymbol{E}_{\boldsymbol{b}}(\boldsymbol{k}+\mathbf{1})=\boldsymbol{E}_{\boldsymbol{b}}(\boldsymbol{k})-h(k) V_{o c} \tilde{\boldsymbol{i}}(\boldsymbol{k}) .
\end{gathered}
$$

The cell current $i(k) \in\left[i_{\min }, i_{\max }\right]$ is chosen to be positive when discharging. The current $\tilde{\boldsymbol{i}}(\boldsymbol{k})=\boldsymbol{s}_{\text {bat }} t(k)$ is a variable change that is introduced to replace the non-convex product of two variables $\left(\boldsymbol{s}_{\boldsymbol{b a t}} i(k)\right)$ by a quadratic-over-linear function $\left(\frac{\tilde{i}^{2}(k)}{s_{b a t}}\right)$ which is convex in both $\tilde{\boldsymbol{i}}(\boldsymbol{k})$ and $\boldsymbol{s}_{\text {bat }}$. It is clear that the battery model does not depend on the configuration of the cells (series/parallel), but rather on the total number of cells. The voltage over each cell, $V_{o c}$, is in practice a function of the $\mathrm{SoC}$ of the battery; however, in this study it is approximated to be constant. This assumption can be justified when operating in limited SoC ranges, as illustrated in Fig. 6 .

During the available parking periods, $\Delta t_{c}(k)$, it is assumed without loss of generality that the vehicle is charged 
with constant current and power. Then, to save computation time by not simulating over the many charging samples during a charging period, it is assumed that the whole charging energy enters the battery in one extra long sample, $\Delta t_{c}(k)$, at the parking events. In this way, at charging occasions in $(11), h(k)$ is equal to $\Delta t_{c}(k)$.

\section{Electric motor}

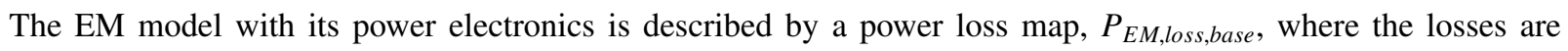
measured at steady-state for different torque-speed combinations. The power losses for each EM speed can be approximated by a second-order polynomial in the torque

$$
P_{E M, l o s s, \text { base }}\left(\omega_{E M}, T_{E M, \text { base }}\right)=c_{1}\left(\omega_{E M}\right) T_{E M, \text { base }}^{2}+c_{2}\left(\omega_{E M}\right) T_{E M, \text { base }}+c_{3}\left(\omega_{E M}\right)
$$

where the coefficients $c_{1} \geq 0, c_{2}$ and $c_{3}$ are functions of $\omega_{E M}$ and therefore are time dependent $(c(k))$. The accuracy of this approximation is high and is discussed in [14]. The coefficients are calculated using least squares method for grids over $\omega_{E M}$ to fit the polynomial in (12) to the measured data. For speed values not belonging to the grid nodes, the coefficients are obtained by linear interpolation.

To vary the size of the EM, the maximum and minimum torque, $T_{E M, \text { max base }}\left(\omega_{E M}(k)\right)$ and $T_{E M, \text { min,base }}\left(\omega_{E M}(k)\right)$, are scaled by a scaling factor $\boldsymbol{s}_{\boldsymbol{E}}$ [11]. The losses are assumed to change linearly with $\boldsymbol{s}_{\boldsymbol{E} \boldsymbol{M}}$ [21]. Hence, given a baseline EM described by torque $T_{\text {em,base }}$ and losses $P_{E M, l o s s, b a s e}$, the losses of the scaled EM are calculated at each time instant as

$$
\begin{aligned}
P_{E M, \text { loss }}(k) & =\boldsymbol{s}_{\boldsymbol{E} \boldsymbol{M}} P_{E M, \text { loss,base }}(k) \\
& =\boldsymbol{s}_{\boldsymbol{E} \boldsymbol{M}}\left(c_{1}(k) T_{E M, \text { base }}^{2}(k)+c_{2}(k) T_{E M, \text { base }}(k)+c_{3}(k)\right) \\
& =\boldsymbol{s}_{\boldsymbol{E} \boldsymbol{M}}\left(c_{1}(k)\left(\frac{\boldsymbol{T}_{\boldsymbol{E M}}(\boldsymbol{k})}{\boldsymbol{s}_{\boldsymbol{E} \boldsymbol{M}}}\right)^{2}+c_{2}(k) \frac{\boldsymbol{T}_{\boldsymbol{E M}}(\boldsymbol{k})}{\boldsymbol{s}_{\boldsymbol{E M}}}+c_{3}(k)\right) \\
& =c_{1}(k) \frac{\boldsymbol{T}_{\boldsymbol{E M}}^{\mathbf{2}}(\boldsymbol{k})}{\boldsymbol{s}_{\boldsymbol{E M}}}+c_{2}(k) \boldsymbol{T}_{\boldsymbol{E M}}(\boldsymbol{k})+c_{3}(k) \boldsymbol{s}_{\boldsymbol{E M}} .
\end{aligned}
$$

This nonlinear model is convex in $\boldsymbol{T}_{\boldsymbol{E} \boldsymbol{M}}$ and $\boldsymbol{s}_{\boldsymbol{E} \boldsymbol{M}}$ for $\boldsymbol{s}_{\boldsymbol{E} \boldsymbol{M}}>0$.

\section{Internal combustion engine}

The fuel power, $P_{f}$, of an ICE is a function of the engine torque and the engine speed and is derived from a map obtained from engine experiments at steady state. The fuel power represented by Willan's lines [19], [20], can be approximated for each engine speed with a second-order polynomial in $T_{I C E}$ as

$$
P_{f, \text { base }}\left(\omega_{I C E}, T_{I C E, \text { base }}\right)=b_{1}\left(\omega_{I C E}\right) T_{I C E, \text { base }}^{2}+b_{2}\left(\omega_{I C E}\right) T_{I C E, \text { base }}+b_{3}\left(\omega_{I C E}\right)
$$

where the coefficients $b_{1} \geq 0, b_{2}$, and $b_{3}$ are functions of $\omega_{I C E}$ and hence time dependent $(b(k))$, and are calculated in a similar way as $c_{1}, c_{2}$, and $c_{3}$ for the EM. The accuracy of this approximation is also discussed in [14].

To vary the ICE size, linear torque scaling is used [21], [13], which means that the torque is given by

$$
\boldsymbol{T}_{I C E}\left(\omega_{I C E}(k)\right)=\boldsymbol{s}_{I C E} T_{I C E, b a s e}\left(\omega_{I C E}(k)\right),
$$


where $\boldsymbol{s}_{I C E}$ is the scaling factor. This approach is more accurate if the size is not very far from the baseline size and for an ICE with fixed number of cylinders; therefore, we limit the range of the $\boldsymbol{S}_{\boldsymbol{I C E}}$. An alternative solution would be to update the engine map using engine simulators like TDES [22], [23] and solve the optimization again, in case the optimal engine size is not close to the nominal value. Using linear scaling, the losses scale linearly, i.e., the efficiency of the ICE $\eta_{I C E}$ is

$$
\eta_{I C E}\left(\omega_{I C E}, \boldsymbol{T}_{I C E}\right)=\eta_{I C E, b a s e}\left(\omega_{I C E}, \frac{\boldsymbol{T}_{I C E}}{\boldsymbol{S}_{I C E}}\right)
$$

In this way, using (14), the fuel power is calculated as

$$
\begin{aligned}
& P_{f}(k)=\frac{\omega_{I C E}(k) \boldsymbol{T}_{\text {ICE }}(\boldsymbol{k})}{\eta_{I C E}\left(\omega_{I C E}, \boldsymbol{T}_{\text {ICE }}\right)} \\
& =\frac{\omega_{I C E}(k) \boldsymbol{s}_{I C E} T_{I C E, \text { base }}}{\eta_{I C E, \text { base }}\left(\omega_{I C E}, \frac{\boldsymbol{T}_{\text {ICE }}}{\boldsymbol{s}_{\text {ICE }}}\right)} \\
& =\boldsymbol{s}_{I C E} P_{f, \text { base }}(k) \\
& =s_{I C E}\left(b_{1}(k) T_{I C E, \text { base }}^{2}(k)+b_{2}(k) T_{\text {ICE, base }}(k)+e_{\text {on }}(k) b_{3}(k)\right) \\
& =\boldsymbol{s}_{\boldsymbol{I C E}}\left(b_{1}(k)\left(\frac{\boldsymbol{T}_{\boldsymbol{I C E}}(\boldsymbol{k})}{\boldsymbol{s}_{\boldsymbol{I C E}}}\right)^{2}+b_{2}(k) \frac{\boldsymbol{T}_{\boldsymbol{I C E}}(\boldsymbol{k})}{\boldsymbol{s}_{\boldsymbol{I C E}}}+e_{\text {on }}(k) b_{3}(k)\right) \\
& =b_{1}(k) \frac{\boldsymbol{T}_{\boldsymbol{I C E}}^{2}(\boldsymbol{k})}{\boldsymbol{s}_{\boldsymbol{I C E}}}+b_{2}(k) \boldsymbol{T}_{\boldsymbol{I C E}}(\boldsymbol{k})+e_{o n}(k) b_{3}(k) \boldsymbol{s}_{\boldsymbol{I C E}} .
\end{aligned}
$$

The reason for introducing the variable $e_{o n}$ is to remove the idling losses $b_{3}$, when the ICE is off. The decision for turning the ICE on, $e_{o n}(k)=1$, or off, $e_{o n}(k)=0$, has to be made prior to the optimization to preserve the problem convexity and it is decided based on the baseline power demand required by the vehicle when following the driving cycle, $P_{\text {dem,base }}(k)$. The baseline mass, $m$, used to calculate $P_{\text {dem,base }}(k)$, is the mass of the vehicle with the baseline component sizes and it is given in Table III. At every time instant, if the power demand is higher than a power threshold, $P_{o n}$, the ICE is turned on and is turned off otherwise. This is shown as

$$
e_{o n}(k)= \begin{cases}1 & \text { if } P_{\text {dem,base }}(k)>P_{o n} \\ 0 & \text { otherwise. }\end{cases}
$$

A good value of $P_{\text {on }}$ can be chosen so that the engine is on $95 \%$ of the times (see [24]). We can also iterate the optimization over several values of $P_{\text {on }}$ to find the best result.

\section{E. Cost and mass model}

Finally, we need to define how the costs and masses of the battery, EM, and ICE depend on the scaling parameters. Linear cost and weight models are used by many authors, e.g., in [25] and [26], and give accurate estimates as long as the optimal sizes are of the same order of magnitude as the baseline $(50 \%-200 \%)$ :

$$
\begin{aligned}
\operatorname{cost}_{j} & =\operatorname{cost}_{j, i}+\operatorname{cost}_{j, s} s_{j} \\
m_{j} & =m_{j, s} s_{j}
\end{aligned}
$$

where $j \in\{$ bat, EM,ICE $\}$ and the values for $\operatorname{cost}_{j, i}$, cost $_{j, s}$ and $m_{j, s}$ are given in Table IV. The total mass of the 
TABLE IV

PARAMETERS OF THE COST AND MASS MODEL

\begin{tabular}{ll||ll}
\hline $\operatorname{cost}_{\text {bat }-i}$ & $€ 500$ & $m_{\text {bat }, s}$ & $0.687 \mathrm{~kg}$ \\
$\operatorname{cost}_{\text {bat }-s}$ & $€ 63.7$ & & \\
\hline $\operatorname{cost}_{E M-i}$ & $€ 1750$ & $m_{E M, s}$ & $48 \mathrm{~kg}$ \\
$\operatorname{cost}_{E M-s}$ & $€ 875$ & & \\
\hline $\operatorname{cost}_{I C E-i}$ & $€ 575$ & $m_{I C E, s}$ & $97.5 \mathrm{~kg}$ \\
$\operatorname{cost}_{I C E-s}$ & $€ 666.7$ & & \\
\hline
\end{tabular}

vehicle, $m_{t o t}$, is therefore calculated as

$$
m_{t o t}=m_{g}+m_{b a t}+m_{E M}+m_{I C E}
$$

where $m_{g}$ is the glider mass (the vehicle minus its drivetrain) given in Table III. The cost and mass functions are calculated from a baseline EM of $35 \mathrm{~kW}$ power, and a baseline ICE with a displacement size equal to 1.6 liter and power equal to $65 \mathrm{~kW}$. The battery is energy optimized with cell capacity of $Q=159 \mathrm{Wh}$. It is important to notice that the choice of the battery type will influence the result of the optimization (see [27] and [28]).

\section{CONVEX OPTIMIZATION PROBLEM}

In this section, the problem formulated in Section II is presented in more details. The optimization problem is convex, which in its general form can be written as

$$
\begin{array}{cl}
\inf _{x} & f_{0}(x) \\
\text { subject to } & f_{i}(x) \leq 0, i=1, \ldots, m, \\
& h_{j}(x)=0, j=1, \ldots, p,
\end{array}
$$

where the cost function $f_{0}(x)$ and the constraints $f_{1}, \ldots, f_{m}: R^{n} \rightarrow R$ are convex and $h_{1}, \ldots, h_{n}$ are affine functions. The cost to be minimized in the optimization problem includes the operational and component costs for a vehicle driven on a cycle. The decision variables, $x$, to be found by the optimization solver are $\boldsymbol{s}_{\boldsymbol{b a t}}, \boldsymbol{s}_{\boldsymbol{I C E}}, \boldsymbol{s}_{\boldsymbol{E} \boldsymbol{M}}$ which are scalars and $\boldsymbol{T}_{I C E}^{N}, \boldsymbol{T}_{E M}^{N}, \tilde{i}^{N}, P_{b r k}^{N}, E_{b}^{N+1}, P_{g}^{N_{c}}$ which are vectors. The superscripts show the length of the vectors, where $N$ is the number of time samples of the driving cycle. In our problem, there are slightly more than 300000 variables, which are found by the solver in one round of optimization in about 25 minutes 5 .

As mentioned earlier, the constraints $f_{1}, \ldots, f_{m}$ and $h_{1}, \ldots, h_{n}$ in (21) include the equations governing the power flow in the system, i.e., (6) and (7), the component models, i.e., (10), (11), (13), (17), (19), and the limitations of the components. Recall that, for a convex problem in (21), nonlinear functions must not be tied with equalities.

\footnotetext{
${ }^{5} \mathrm{On}$ a standard PC with 4GB RAM and $2.66 \mathrm{GHz}$.
} 
TABLE V

CONVEX PROBLEM For THE PARALLEL PHEV

\begin{tabular}{|c|c|}
\hline Variables & $T_{I C E}^{N}, T_{E M}^{N}, P_{b r k}^{N}, \tilde{i}^{N}, E_{b}^{N+1}, P_{g}^{N_{c}}, s_{b a t}, s_{I C E}, s_{E M}$ \\
\hline minimize & $\operatorname{cost}()$. \\
\hline \multirow[t]{14}{*}{ subject to } & $P_{d e m}(k)-\boldsymbol{P}_{\boldsymbol{b r} \boldsymbol{k}}(\boldsymbol{k})=\boldsymbol{T}_{\boldsymbol{E} \boldsymbol{M}}(\boldsymbol{k}) \omega_{E M}(k)+e_{o n}(k) \boldsymbol{T}_{\boldsymbol{I C E}}(\boldsymbol{k}) \omega_{I C E}(k) \eta(k)$ \\
\hline & $\omega_{E M}(k) \boldsymbol{T}_{\boldsymbol{E}} \boldsymbol{M}(\boldsymbol{k})+P_{E M, l o s s}(k)+P_{a u x}(k) \leq P_{b a t}(k)+\boldsymbol{P}_{\boldsymbol{g}}(\boldsymbol{k}) \eta_{g}$ \\
\hline & $P_{E M, l o s s}\left(\boldsymbol{T}_{\boldsymbol{E M}}(\boldsymbol{k}), \boldsymbol{s}_{\boldsymbol{E} \boldsymbol{M}}\right)=c_{1}(k) \frac{\boldsymbol{T}_{\boldsymbol{E M}}^{2}(\boldsymbol{k})}{\boldsymbol{s}_{\boldsymbol{E M}}}+c_{2}(k) \boldsymbol{T}_{\boldsymbol{E M}}(\boldsymbol{k})+c_{3}(k) \boldsymbol{s}_{\boldsymbol{E M}}$ \\
\hline & $P_{b a t}\left(i(k), s_{b a t}\right)=V_{o c} \tilde{i}(k)-R \frac{\tilde{i}^{2}(k)}{s_{b a t}}$ \\
\hline & $E_{b}(2: k+1)=E_{b}(1: k)-h(k) \tilde{i}(1: k) V_{o c}$ \\
\hline & $\boldsymbol{T}_{\boldsymbol{E M}}(\boldsymbol{k}) \in\left[T_{E M, \text { min,base }}\left(\omega_{E M}(k)\right), T_{E M, \text { max }, \text { base }}\left(\omega_{E M}(k)\right)\right] \boldsymbol{s}_{\boldsymbol{E M}}$ \\
\hline & $\boldsymbol{T}_{I C E}(\boldsymbol{k}) \in\left[0, \boldsymbol{s}_{\boldsymbol{I C E}} T_{I C E, \text { max }, \text { base }}\left(\omega_{I C E}(k)\right)\right]$ \\
\hline & $\tilde{\boldsymbol{i}}(k) \in\left[i_{\min }, i_{\text {max }}\right] \boldsymbol{s}_{\text {bat }}$ \\
\hline & $\boldsymbol{E}_{\boldsymbol{b}}(\boldsymbol{k}) \in\left[S o C_{\min }, S o C_{\max }\right] V_{o c} Q$ \\
\hline & $\boldsymbol{P}_{\boldsymbol{g}}(\boldsymbol{k}) \in\left[0, P_{g, \max }(k)\right]$ \\
\hline & $s_{\text {bat }} \in\left[s_{\text {bat }, \min }, s_{\text {bat }, \max }\right]$ \\
\hline & $\boldsymbol{s}_{E \boldsymbol{M}} \in\left[s_{E M, \min }, s_{E M, \max }\right]$ \\
\hline & $\boldsymbol{s}_{\text {ICE }} \in\left[s_{I C E, \min }, s_{I C E, \max }\right]$ \\
\hline & $\forall k \in\{0, \ldots, N-1\}$ \\
\hline
\end{tabular}

Clearly using (10) and (13) in (7) yields a nonlinear equality constraint. Therefore, the equality is relaxed to an inequality as

$$
\omega_{E M}(k) \boldsymbol{T}_{\boldsymbol{E M}}(\boldsymbol{k})+P_{E M, l o s s}(k) \leq P_{b a t}(k)-P_{a u x} .
$$

It is easy to see that at the optimum, the constraint will be satisfied with equality since otherwise the battery will throw away energy unnecessarily.

Moreover, to keep the problem convex, $\boldsymbol{s}_{\boldsymbol{b} \text { at }}$ is relaxed to a real value. The relaxation will introduce a rounding error that has a small influence on the optimal result. This is because either the cell capacity can be considered very small to give large number of cells, or the result can be interpreted as an indication of the optimal pack capacity. The convex problem, shown in Table V, is automatically translated by a tool CVX [29], to a form required by a publicly available solver, Sedumi [30].

\section{Numerical Results}

In this section, we give results of the simultaneous optimization of energy management and component sizing over the driving cycle described previously. Different performance requirements are considered, either as constraints on the component sizes or via an appended performance cycle as described in Section II.

1) Performance requirements: In Table VI the results of the optimization for the real-life driving cycle, with and without considering the baseline performance requirements $\left(\alpha_{p}=1, v_{\max }=120 \mathrm{kph}\right.$ and no AER requirements) are shown. The values in the table show that the performance requirements have a high influence on the size of the components and the costs. The main difference is in the size of the ICE. This is mainly because the battery cells are 
TABLE VI

OPTIMAL COSTS AND COMPONENT SIZES FOR THE DRIVING CYCLE, WITH AND WITHOUT CONSIDERING THE PERFORMANCE REQUIREMENTS (PR)

\begin{tabular}{lll}
\hline \hline variables & with PR & without PR \\
\hline battery capacity & $5.3 \mathrm{kWh}$ & $6.4 \mathrm{kWh}$ \\
EM power & $32.5 \mathrm{~kW}$ & $32.5 \mathrm{~kW}$ \\
ICE power & $128.1 \mathrm{~kW}$ & $61.6 \mathrm{~kW}$ \\
fuel cost & $€ 22.1$ & $€ 17.4$ \\
electricity cost & $€ 5.8$ & $€ 6.1$ \\
component costs & $€ 32.3$ & $€ 31.0$ \\
total cost & $€ 60.2$ & $€ 54.7$ \\
\hline
\end{tabular}

energy optimized with very limited power, and since during the performance cycle the power demand is high, the number of battery cells must be large. On the other hand a heavy and large capacity battery is not needed in the rest of the driving cycle. Therefore, the optimization finds it cheaper to provide the power for the performance cycle by the ICE. Due to a larger ICE and smaller battery capacity, the fuel consumption increases and the electricity usage decreases. In Fig. 7, the driving cycle (without the performance cycle) and the optimal SoC trajectory are shown.
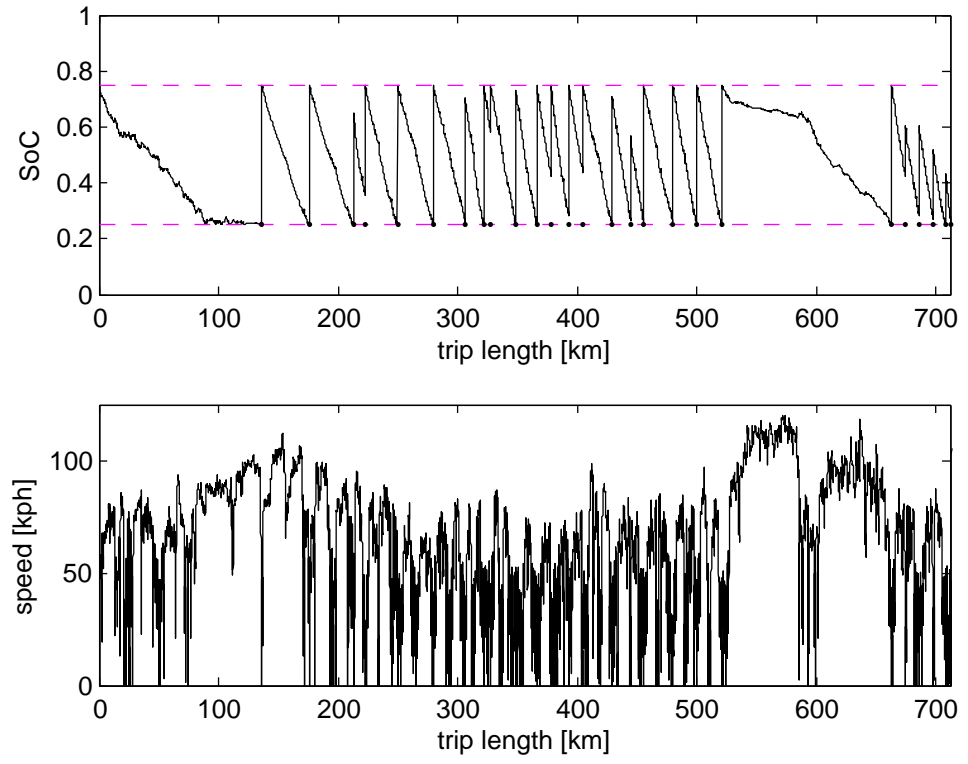

Fig. 7. Optimal SoC trajectory; the battery has the possibility to be charged from the grid at positions shown by dot (upper plot) and demanded speed profile (lower plot).

To see the effect of different levels of performance requirements, the optimization is done over driving cycles including performance cycles with $\alpha_{p}=[0.7,0.8,0.9,1.0,1.1,1.2]$ and top speed of $v_{\max }=120$ and $150 \mathrm{kph}$. The result of the optimization is shown in Figs. 8 and 9. In Fig. 8 the power of the EM and the ICE, and the 0 $100 \mathrm{kph}$ acceleration time are plotted vs. $\alpha_{p}$. For high values of $\alpha_{p}$, the power of the ICE increases noticeably, 

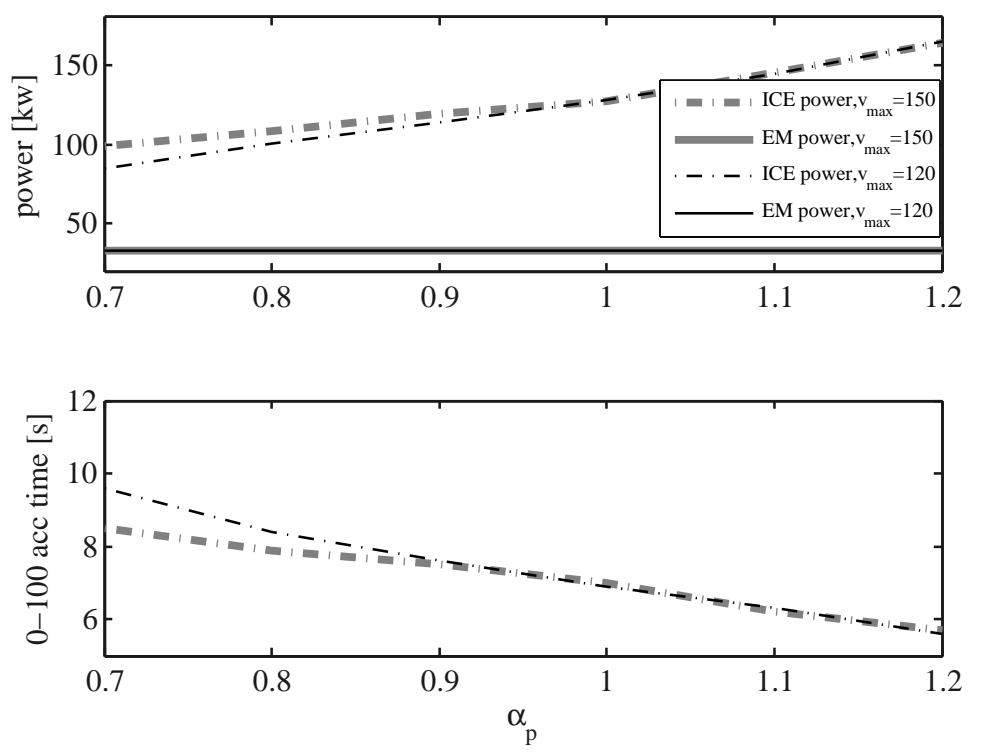

Fig. 8. Power of ICE and EM for top speeds of $120 \mathrm{kph}$ (thin line) and $150 \mathrm{kph}$ (thick line) vs. $\alpha_{p}$ (upper plot) and 0 to $100 \mathrm{kph}$ acceleration time vs. $\alpha_{p}$ (lower plot).

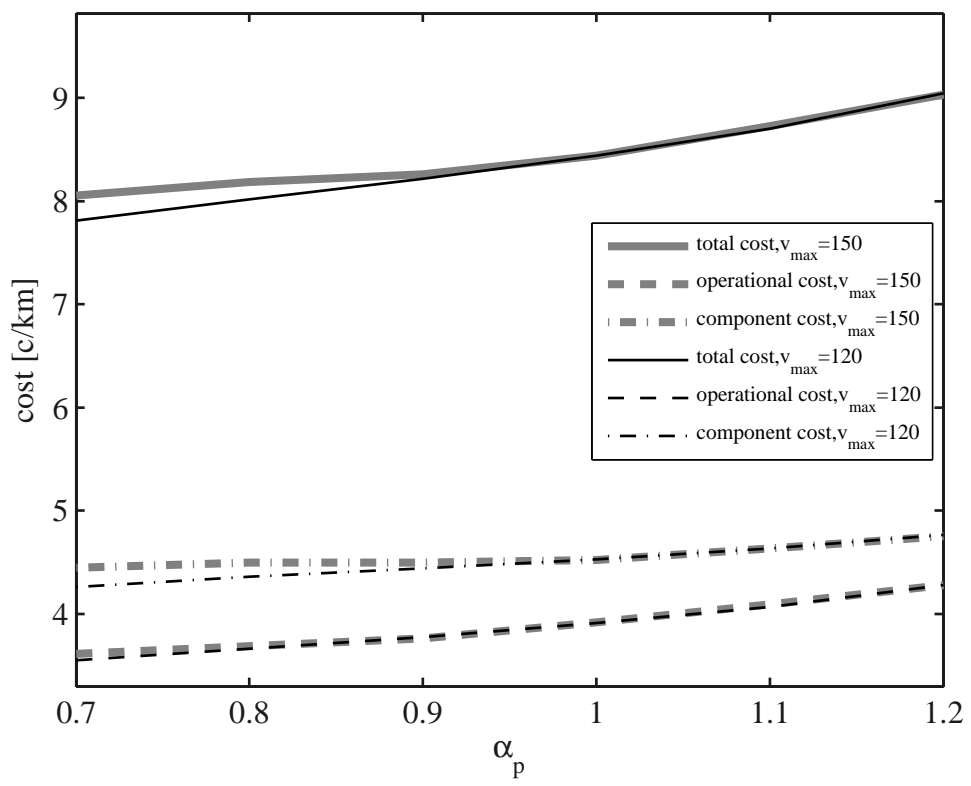

Fig. 9. Total cost, component costs and operational cost vs. $\alpha_{p}$, for top speeds of $120 \mathrm{kph}$ (thin line) and $150 \mathrm{kph}$ (thick line).

whereas the power of the EM does not change. Again, this is because of the type of the battery. On the other hand, the top speed demand only affects the ICE size for small values of $\alpha_{p}$, and for larger values of $\alpha_{p}$, the ICE is sized by the acceleration requirements and it does not change for different top speed requirements. Clearly, increasing 
the acceleration demand decreases the resulting $0-100 \mathrm{kph}$ acceleration time. This plot is an indication of the performance of the vehicle. Fig. 9 shows that higher acceleration and top speed requirements increase both the components and operational costs. The increase in the operational cost is because larger ICEs have more working points at lower efficiency (compared to smaller ICEs) and hence higher fuel consumption. In the figures, costs are given in euro cent per kilometer $[\mathrm{c} / \mathrm{km}]$, for easier comparison.

Another requirement on PHEVs is the AER which is directly related to the size of the battery. To see the effect of this requirement, the optimization is done with different fixed battery sizes. For all the cases, the acceleration and top speed requirements are kept constant as $v_{\max }=120 \mathrm{kph}$ and $\alpha_{p}=1$. The results in Fig. 10 show that increasing the battery capacity decreases the operational cost, but on the other hand, the component costs increases linearly. The total cost decreases until it reaches the optimal battery size, here $5.2 \mathrm{kWh}$, which is equivalent to approximately $20 \mathrm{~km}$ of AER. For larger battery sizes, the increase in the components cost is more than the gain in the operational cost, and for very large batteries, the operational cost starts increasing due to the increase in the battery mass. However, the curve is rather flat between 15 to $35 \mathrm{~km} \mathrm{AER,} \mathrm{which} \mathrm{is} \mathrm{because} \mathrm{low} \mathrm{AER} \mathrm{leads} \mathrm{to} \mathrm{low}$ vehicle cost but higher operational cost and vice versa. This means that in this range of AER, the cost is not very sensitive to the requirement on AER.

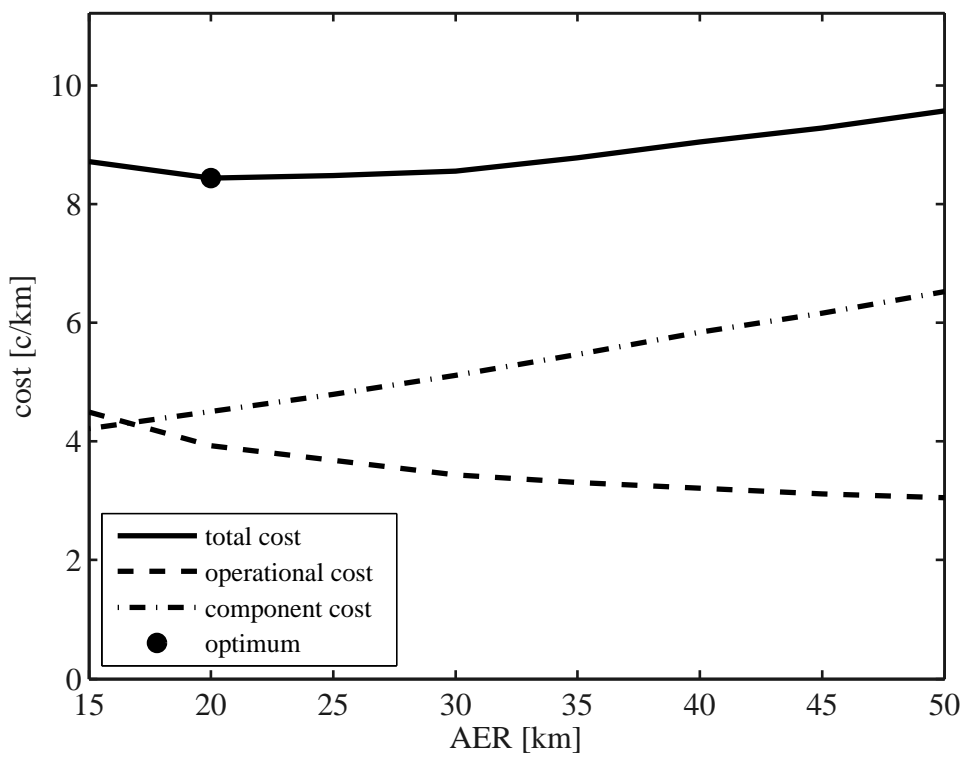

Fig. 10. Components, operational and total optimal cost (euro cents per kilometer [c/km]) vs. AER; the minimum cost is shown with a circle.

2) Battery and fuel price: To study the effect of change in battery and fuel price, the optimization is done for three different cases; in the first case, the prices of the electricity and battery are the present prices, i.e., $\rho_{f}=1.34 \mathrm{EUR} / \mathrm{L}$ and cost $_{\text {bat }}=400 \mathrm{EUR} / \mathrm{kWh}$. In the second case, the optimization includes $30 \%$ cheaper battery price and in the third case, 30\% more expensive fuel price. As shown in Fig. 11, in the last two cases, the optimal AER is around 
$30 \mathrm{~km}$, which means that it is optimal to have a large AER for higher fuel cost or cheaper battery price. It can also be observed that with the current price, a vehicle with $50 \mathrm{~km}$ AER costs $14 \%$ more than the optimal design, whereas for the other two cases, the difference is only $5 \%$.

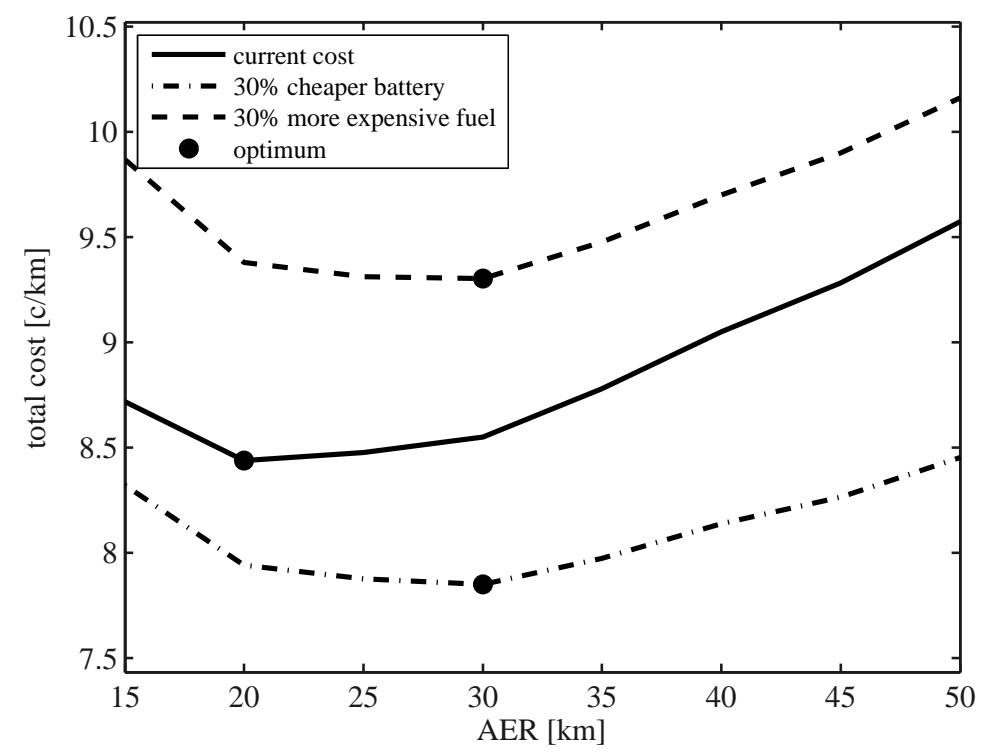

Fig. 11. Total cost with the present battery and fuel cost, $30 \%$ cheaper batteries and $30 \%$ more expensive fuel; the minimum costs are shown with circles.

3) Pareto front: The value of the weighting factor $w_{\text {comp }}$ in (3) is defined for a fixed lifetime and yearly driving length. However, in real-life, this value is different for different vehicles. By varying $w_{\text {comp }}$, a Pareto front is obtained for the optimal operational cost vs. the optimal component costs; this is shown in Fig. 12 for different acceleration requirements. The figure shows that component and operational costs are conflicting objectives as expected. In other words, components that are more expensive can lower the operational cost. The component costs increases mainly due to increase in the battery and EM size, which clearly reduces fuel consumption. From (3) we can see that for a vehicle with a longer lifetime driving distance, the value of $w_{\text {comp }}$ is smaller, and therefore, it is optimal to pay more for the components and get a lower operational cost, compared to a vehicle with a shorter lifetime driving distance. Naturally, higher performance requirements increase both the component and operational costs. 


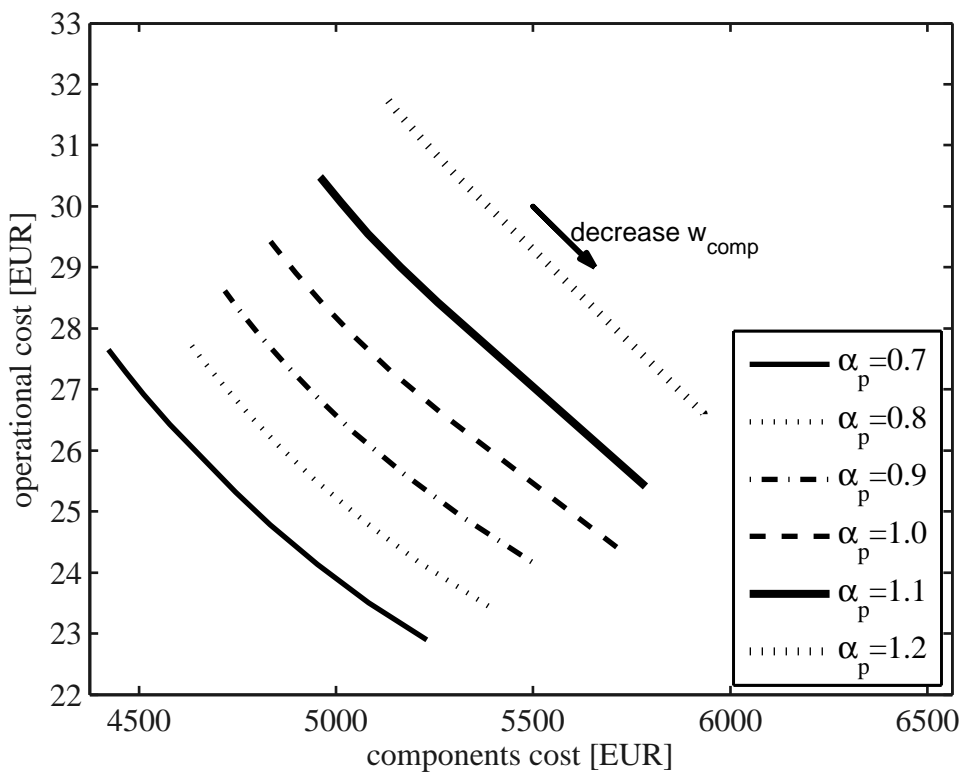

Fig. 12. Set of Pareto points using different weighting factors between operational cost and component costs.

\section{Conclusion}

In this paper, a new method of simultaneous optimization of components sizes (for battery, electric motor, and internal combustion engine) and energy management (torque split and charging) for a parallel plug-in hybrid electric vehicle is introduced. The gear and engine on-off is found by heuristics, and the remaining sub-problem is formulated as a convex optimization problem by approximating the quasi-static models with nonlinear convex functions. The convex formulation allows fast solutions of large-size problems, giving short turn-around design cycles. The method has also the advantage of handling very long driving cycles, which is necessary to find optimal component sizes that are robust to the varying real-life driving cycles.

The result of the optimization depends on many factors, e.g., performance requirements, charging behavior, driving cycle, battery type, energy and component costs, and gear shifting. The method is used to study the effect of these factors on the design of a PHEV. In particular, the effect of the performance requirements on the sizing of vehicle components is studied. For the examples shown in the paper, the results indicate that the vehicle cost is more affected by the acceleration requirements, than the requirements on top speed and all electric range. This means that performance requirements have to be included in the optimization problem, otherwise the result will be misleading. Moreover, with the current price of energy and battery cells, it is not optimal to have very long AER for PHEVs.

In general, the method can be used as a tool to give a better understanding of the influence of different factors on vehicle design. In addition, current work addresses the limitation that is posed by the need to fix the gears and engine on-off prior to the optimization. 


\section{AcKNowledgment}

The authors are grateful to Dr. Lars Johannesson for fruitful discussions, and to reviewers for many suggestions to improve the readability of the paper. This work was supported by the Swedish Energy Agency. The simulations were performed on resources provided by the Swedish National Infrastructure for Computing (SNIC) at C3SE.

\section{REFERENCES}

[1] T. C. Moore, "Tools and strategies for hybrid-electric drivesystem optimization," SAE Tech. Paper 961660, 1996, doi:10.4271/961660.

[2] P. Valdes-Dapena (2007, May), We want better mileage - but power and size, too, [Online]. Available: http: //www.CNNMoney.com

[3] T. Miller, G. Rizzoni and Q. Li, "Simulation-based hybrid-electric vehicle design search," SAE Tech. Paper 1999-01-1150, 1999, doi: 10.4271/1999-01-1150.

[4] O. Sundström, L. Guzzella and P. Soltic, "Torque-assist hybrid electric powertrain sizing: from optimal control toward optimal sizing law," IEEE Trans. Control Syst. Technol., vol. 18, no. 4, 2010.

[5] L. Wu, Y. Wang, X. Yuan and Z. Chen, "Multiobjective optimization of HEV fuel economy and emissions using the self-adaptive differential evolution algorithm," IEEE Trans. Veh. Technol., vol. 60, no. 6, Jul. 2011.

[6] X. Hu, Z. Wang, L. Liao, "Multi-Objective optimization of HEV fuel economy and emissions using evolutionary computation," SAE Tech. Paper 2004-01-1153, 2004, doi: 10.4271/2004-01-1153.

[7] V. Galdi, L. Ippolito, A. Piccolo, and A. Vaccaro, "A genetic-based methodology for hybrid electric vehicles sizing," Soft Computing, vol. 5, no. 6, pp. 451-457, 2009.

[8] M. Montazeri-Gh and A. Poursamad, "Application of genetic algorithm for simultaneous optimisation of HEV component sizing and control strategy," Int. J. Alternative Propulsion, vol. 1, no. 1, 2006.

[9] J. Hellgren and B. Jacobson, “A systematic way of choosing driveline configuration and sizing components in hybrid vehicles," SAE Tech. Paper 2000-01-3098, 2000, doi:10.4271/2000-01-3098.

[10] R. Fellini, N. Michelena, P. Papalambros, and M. Sasena “Optimal design of automotive hybrid powertrain systems," Proc. First Int. Symp. on Environmentally Conscious Design and Inverse Manufacturing, Los Alamitos, CA, USA, pp. 400-405, 1-3 Feb. 1999.

[11] C. S. N. Shiau, N. Kaushal, C. T. Hendrickson, S. B. Peterson, J. F. Whitacre, and J. J. Michalek, "Optimal Plug-In Hybrid Electric Vehicle Design and Allocation for Minimum Life Cycle Cost,Petroleum Consumption, and Greenhouse Gas Emissions,” J. Mech. Design, vol. 132, pp. 091013 (11), 2010.

[12] S. J. Mouraa, D. S. Callawayb, H. K. Fathya, and J. L. Steina, "Tradeoffs between battery energy capacity and stochastic optimal power management in plug-in hybrid electric vehicles," J. Power Sources, vol. 195 , no. 9, pp. 2979-2988, 2009.

[13] Z. S. Filipi, L. S. Louca, B. Daran, C. C. Lin, U. Yildir, B. Wu, M. Kokkolaras, D. N. Assanis, H. Peng, P. 
Y. Papalambros, and J. L. Stein, "Combined optimization of design and power management of the hydraulic hybrid propulsion system for a 6x6 medium truck," Int. J. Heavy Veh. Sys., vol. 11, pp. 372402, 2004.

[14] N. Murgovski, L. Johannesson, J. Sjöberg, B. Egardt, "Component sizing of a plug-in hybrid electric powertrain via convex optimization,” J. Mechatronics, vol. 22, no. 1, pp. 106-120, Feb. 2012.

[15] Europe's energy portal, [Online]. Available: http://www.energy.eu/

[16] S. Boyd and L. Vandenberghe, Convex optimization, Cambridge University Press, 2009.

[17] L. Guzzella and A. Sciarretta, Vehicle propulsion systems, Springer, 2007.

[18] O. Sundström, L. Guzzella, and P. Soltic, "Optimal hybridization in two parallel hybrid electric vehicles using dynamic programming," Proc. 17th World Congr., The Int. Federation of Automat. Control, Seoul, Korea, Jul. 6-11, 2008.

[19] J.B. Heywood, Internal Combustion Engine Fundamentals, New York: McGraw-Hill, 1988.

[20] S. J. Pachernegg, “A closer look at the Willans-line," SAE Tech. Paper 690182, 1969, doi:10.4271/690182.

[21] O. Sundström, "Optimal control and design of hybrid-electric vehicles," PhD. dissertation, ETH Univ., Zurich, Switzerland, 2009.

[22] D. Assanis and J. Heywood, "Development and Use of a Computer Simulation of the Turbocompounded Diesel System for Engine Performance and Component Heat Transfer Studies," SAE Technical Paper 860329, 1986, doi:10.4271/860329.

[23] D. Assanis, G. Delagrammatikas, R. Fellini, Z. Filipi, J. Liedtke, N. Michelena, P. Papalambros, D. Reyes, D. Rosenbaum, A. Sales, and M. Sasena "An Optimization Approach to Hybrid Electric Propulsion System Design," Mech. Struct. Mach., vol. 27(4), pp. 393-421, 1999.

[24] D., Karbowski, C., Haliburton, A. Rousseau, "Impact of component size on plug-in hybrid vehicles energy consumption using global optimization,” 23rd Int. Electr. Veh. Symp., Anaheim, CA, Dec. 2007.

[25] P. Albertusa, J. Coutsa, V. Srinivasanb, and J. Newmana, "Component sizing optimization of plug-in hybrid electric vehicles," J. Applied Energy, vol. 88, pp. 799804, 2011.

[26] S., Plotkin, and M., Singh, "Multi-Path Transportation Futures Study: Vehicle Characterization and Scenario Analyses," Argonne National Laboratory Report, No. ANL/ESD/09-5.

[27] E. Tara, S. Shahidinejad, S. Filizadeh, and E. Bibeau, "Battery storage sizing in a retrofitted plug-in hybrid electric vehicle," IEEE Trans. Veh. Technol., vol. 59, no. 6, Jul. 2010.

[28] P. Albertusa, J. Coutsa, V. Srinivasan, and J. Newman, "A combined model for determining capacity usage and battery size for hybrid and plug-in hybrid electric vehicles," J. Power Sources, vol. 183, pp. 771782, 2008.

[29] M. Grant and S. Boyd (2010, May), CVX: Matlab software for disciplined convex programming, version 1.21 [Online]. Available: http://cvxr.com/cvx

[30] I. Polik (2010, Jun), Sedumi [Online]. Available: http://sedumi.ie.lehigh.edu/ 


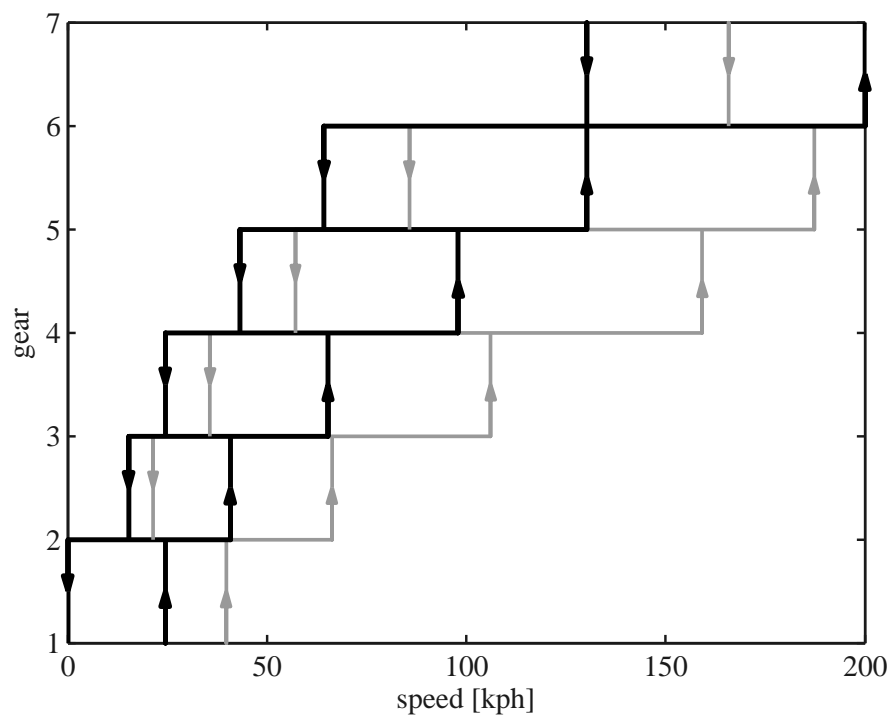

Fig. 13. The speed and power dependent gear shifting strategy.

\section{APPENDIX}

\section{A. Gear selection strategy}

As mentioned before, the gear, $\gamma(k)$, is selected from a speed dependent hysteresis model [18] as shown in Fig. 13. In this strategy, each time the speed exceeds an upper limit, $v_{g u}(\gamma)$, there will be an upshift in the gear and if the speed is lower than a lower limit, $v_{g l}(\gamma)$, there will be a downshift. Knowing the gear ratios, these limits (here called base limits and shown with black lines) are chosen so that the speed of engine is kept in normal operating regions. For instance, $v_{g u}(\gamma)$ is result of a gear strategy where there is an upshift each time the engine speed exceeds $4000 \mathrm{rpm}$ shown as a vertical line, $\omega_{g u}^{b}$, in Fig. 14. However, this strategy highly influences the size of the ICE since it motivates higher torques. Figure 14 shows that for a given power demand if the gear is shifted up in higher speeds, the torque demand will be lower. To lessen this effect, we increase the engine speed threshold at which an upshift occurs, linearly with respect to an acceleration gain, $a_{\text {gain }}=\frac{a(k)}{a_{\left(\alpha_{p}=1\right)}(v(k))}$, for $a_{\text {gain }}>0.7$. This means that for high acceleration demands the gear shifting happens at higher engine speeds. As shown in Fig. 13, the limits change linearly between the base limits $v_{g u}^{b}$, and $v_{g l}^{b}$ (black lines), and peak limits $v_{g u}^{p}$ and $v_{g l}^{p}$ (grey lines) as

$$
\begin{gathered}
v_{g u}(\gamma)= \begin{cases}v_{g u}^{b} & \text { if } a_{\text {gain }}(k)<0.7 \\
\frac{v_{g u}^{p}(\gamma)-v_{g u}^{b}(\gamma)}{1.2-0.7}\left(a_{\text {gain }}(k)-0.7\right)+v_{g u}^{b}(\gamma) & \text { else, }\end{cases} \\
v_{g l}(\gamma)= \begin{cases}v_{g l}^{b} & \text { if } a_{\text {gain }}(k)<0.7 \\
\frac{v_{g l}^{p}(\gamma)-v_{g l}^{b}(\gamma)}{1.2-0.7}\left(a_{\text {gain }}(k)-0.7\right)+v_{g l}^{b}(\gamma) & \text { else. }\end{cases}
\end{gathered}
$$




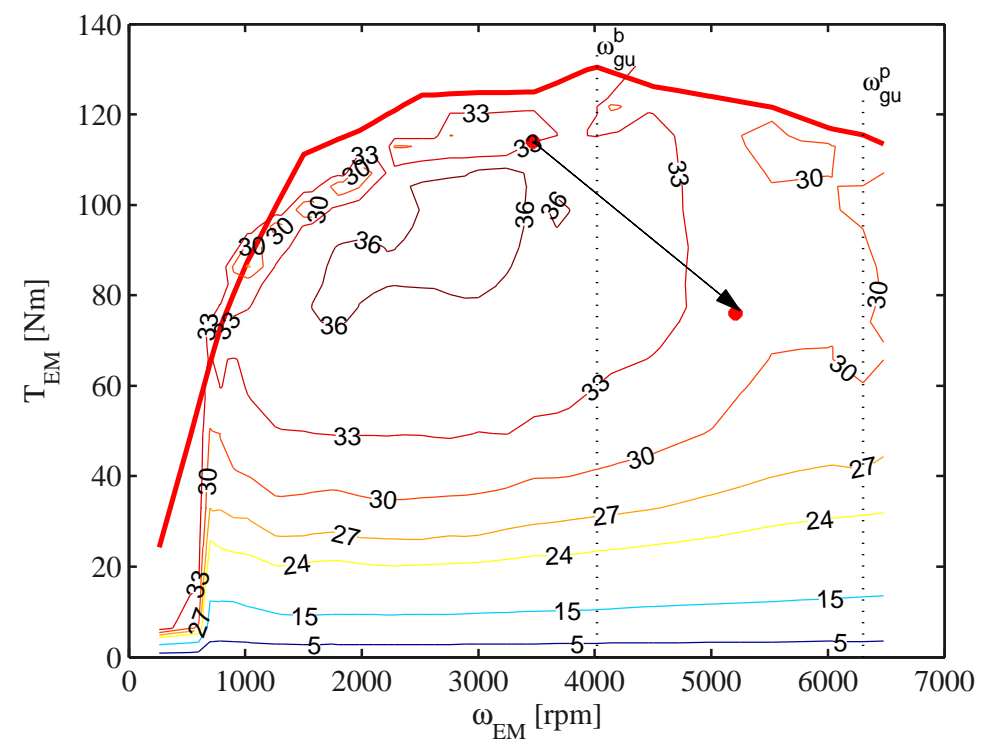

Fig. 14. The baseline limit for changing the gear in low acceleration demand, $\omega_{g u}^{b}$. We can see that for a similar power demand, we get a lower torque if we change the gear at higher speed, $\omega_{g u}^{p}$. The two situations are shown by red markers.

Optimal gear shifting is not considered in this paper, as it may lead to a non-convex problem. Possibilities to improve the gear shifting strategy, by first defining a feasible gear, and then iteratively solving a convex problem and using the result to update the gear selection, will be considered in future studies. 